\title{
Biaxial Tensile Properties of Textured Yarn Fabrics
}

\author{
By Tetsuyuki Sasai* and Sueo Kawabata**, Members, TMSJ \\ *Faculty of Technology, Gunma University, Kiryu, Gunma Prefecture \\ **Department of Polymer Chemistry, Kyoto University, Kyoto
}

Based on Journal of the Textile Machinery Society of Japan, Proceedings, Vol. 36, No. 12, P580-P585 (1983-12)

\begin{abstract}
The capability of predicting the tensile properties of textured yarn fabrics when they are stretched biaxially to equal extension ratios along warp and weft direction is discussed under the tensile force below $500 \mathrm{gf} / \mathrm{cm}$. This force region is related to the handle of fabrics.

Textured yarns are featured with their high stretchability, which makes it difficult to calculate the tensile properties of textured yarn fabrics because the initial extension state of yarns in the fabaric is uncertain. We define this initial extension of yarn, and then calculate the biaxial tensile properties of textured yarn fabrics by applying the biaxial tensile theory by Kawabata et al. Specimens made of nearly flexible yarns showed a good agreement between calculated values and experimentals.
\end{abstract}

\section{Introduction}

The purpose of this study is to discuss the possibility of predicting biaxial tensile properties of textured yarn fabrics made of stretchable textured yarns at relatively low tension below $500 \mathrm{gf} / \mathrm{cm}$ by applying the biaxial tensile theory developed by Kawabata et al. ${ }^{[1]}$

There have been a report on the biaxial tensile properties of fabrics. ${ }^{[3]}$ Kawabata et al. published a biaxial theory of plain fabrics in 1973 using a flexible yarn model and discussed the prediction accuracy of the theory for various spun-yarn fabrics in the wide range of deformation up to breaking point and showed a good agreement between experimental and calculated values. They also applied the biaxial tensile theory on twill fabrics. ${ }^{[2]}$ From these theories, they have found that the amount of thickness decrease of yarns at the intersecting points with the extension of fabrics plays an important role for the calculation of biaxial tensile properties of fabrics.

The specimens used in this experiment are the fabrics made of polyester filament false-twisted textured yarns. Tensile and compressional properties of yarns were measured for those yarns drawn out from the fabrics without giving extension to yarns.

\section{Application of the Biaxial Tensile Theory to Textured Yarn Fabrics}

The highly stretchable property of textured yarns makes their initial extension state in fabric uncertain. Firstly, we have to determine the initial stretched state of the yarn in the unstretched state of fabric. We have defied stretched state of the textured yarn on the basis of the critical extension state of yarns in textured yarn fabrics. ${ }^{[4]}$ There are two methods for this estimation and we have found both of them give the same result. One method is the measurement of extension of yarns drawn out from fabrics, and the other the measure- ment of the apparent yarn diameter in fabrics to estimate the stretched state.

In the former method, we first put two marks at the distance $l_{0}$ on the fabrics. The length of the yarn between the marks in undeformed fabric is denoted by $\widetilde{l}_{0}$ and is measuured directly by the method described later.

We define the weave-crimp ratio $\alpha$ as following:

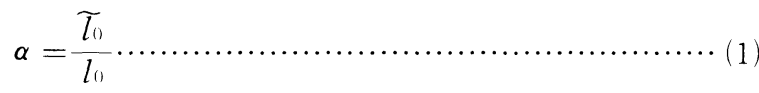

For the textured yarn which has no weave-crimp, we assume the critical extension state of the yarn where only fibercrimps are elongated but fibers in yarn themselves are not elongated. The yarn length at this critical extension state is denoted by $l_{c}$. The value $l_{c}$ can be estimated from the interlacing point of the linear tangential line with the horizontal line in the tensile force-stretch ratio curve of the textured yarn before weaving as shown in Fig. 1.

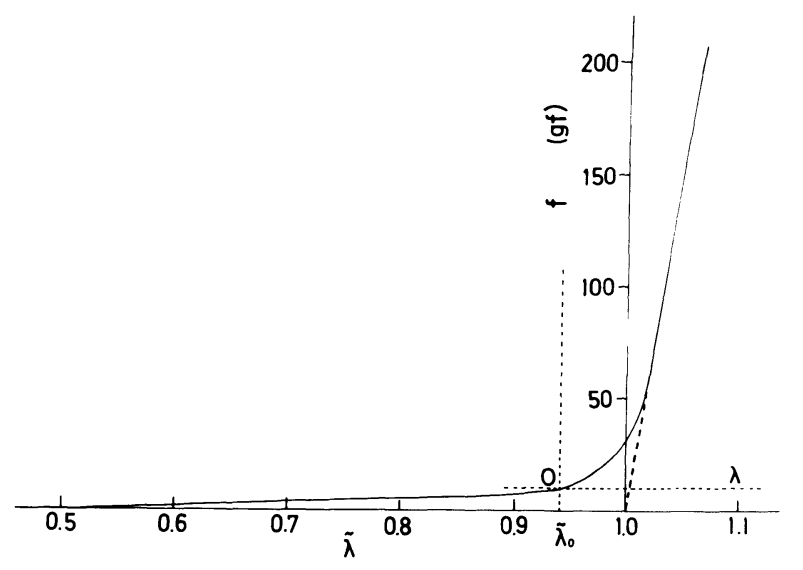

Fig. 1 Load-extension ratio curve of a yarn. The abssissa is normalized by the yarn length at the critical extension state, $l_{c}$. $\lambda_{0}$ is the initial extension state of yarn in the fabric. 
Here, we define yarn stretch ratio $\tilde{\lambda}$ referring $l_{c}$. Then, $\widetilde{\lambda_{0}}$, the initial extension of yarn in the unstretched fabric, is:

$$
\widetilde{\lambda}_{0}=\frac{\tau_{0}}{l_{c}}=\frac{\alpha l_{0}}{l_{c}}
$$

It is easier to measure $\tilde{l_{0}}$ by means of optical measurement from the geometry of the yarn taken out from the fabric instead of the yarn in the fabric. In the case, when the distance between marks will become to be $l_{0}^{\prime}$ when the yarn is drawn out from the fabric, because of the release from restriction of weave structure, we use $\alpha^{\prime}$, alternative to $\alpha$, as defined by the following equation,

$$
\alpha^{\prime}=\frac{\tau_{0}}{l_{0}}
$$

we have from equation (2),

$$
\widetilde{\lambda}_{0}=\frac{\alpha^{\prime} l_{0}^{\prime}}{l_{c}}
$$

The $\widetilde{\lambda_{0}}$ value obtained from equation (2) or (4) gives the initial extension ratio, that is the yarn extension ratio referred to the critial length $l_{c}$ in the fabric in an unstretched state.

The second method to estimate the initial extension state of the yarn in fabric is to measure the apparent yarn diameter in the fabric in the unstretched state to obtain the fiber packing density in the yarn cross-section. In this method, it is necessary to know the relationship between the apparent yarn diameter and yarn extension ratio $\tilde{\lambda}$ (Fig. 2 ).

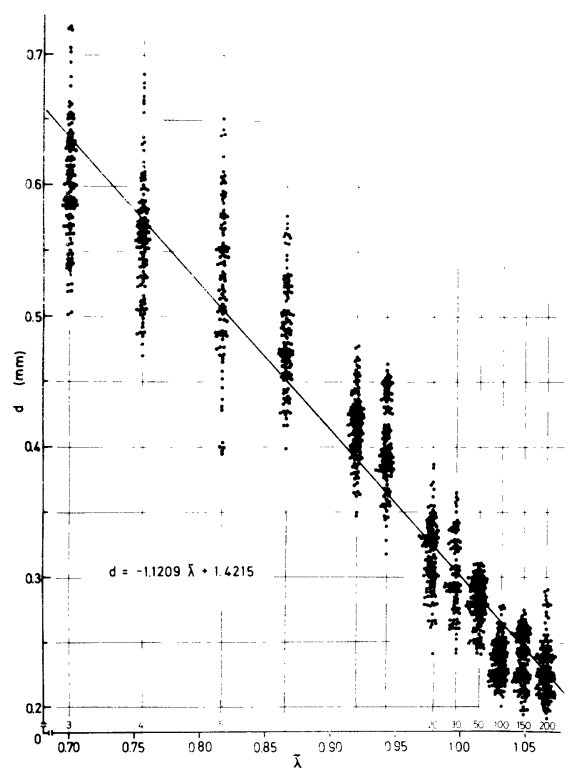

Fig. 2 Relationship between the diameter of the yarn and the extension ratio of yarn.

By the methods above mentioned, we can determine the initial extension state of the yarn in the fabric. Then we can estimate the yarn tensile property which will be substituted in the fabric biaxial tensile theory to obtain the fabric tensile properties by the method as shown in Fig. 5, where the yarn stretch ratio, $\lambda_{y}$, is defined as the stretch ratio referred to the initial stretched state. The horizontal axis of the load-elongation curve of the yarn is changed from $\tilde{\lambda}\left(=l / l_{c}\right)$ referred to the critical length to $\widetilde{\lambda}=\widetilde{\lambda} \times\left(1 / \lambda_{0}\right)$ referred to the length at the initial state in unstretched fabric. ${ }^{[5]}$

\section{Experiments}

3.1 Tensile Property of Yarns Drawn out from the Fabric

Figures 3 and 4 are, respectively, the photographs of surfaces and cross-sections of specimens A, B and C tested in this experiment. Yarn specimens are carefully drawn out from these fabrics not so as to apply so much deformation to yarns. Table 1 shows the yarn extension states, fiber diameters and numbers of fibers in yarns for the three samples.

Yarns in the fabric are in stretched state at the initial extension ratio $\tilde{\lambda}_{0}$ (Fig. 1) when the fabric is unstretched state. This stretching is applied during weaving and related processes. Yarn tensile force is a function of yarn stretch ratio $\lambda_{y}$ and denoted by $g\left(\lambda_{y}\right)$. Here, stretch ratio $\lambda$ is used the value of $\tilde{\lambda} / \widetilde{\lambda_{0}}$ as mentioned before.

Fig. 5 shows the tensile properties of crimped textured yarns drawn out from fabric specimens A, B and C, and also shows how to estimate the yarn tensile property $g\left(\lambda_{y}\right)$. The origin denoted by $\lambda_{y}=1.0$ in the sub-coordinates are the initial extension states mentioned above. We apply the solid line parts to the biaxial tensile theory as yarn tensile property. The yarn tensile properties shown in Fig. 5 are the average properties of ten to twenty measurements of the tensile property. Each curve of the tensile property scatters arround the mean curve and their coefficient of deviation given by (standard deviation)/(mean value) are $9.75 \%, 9.15 \%$, $12.28 \%, 11.48 \%, 8.31 \%$ and $8.75 \%$ in percent for $\mathrm{A} 1, \mathrm{~A} 2$, $\mathrm{B} 1, \mathrm{~B} 2, \mathrm{C} 1$ and $\mathrm{C} 2$, respectively.

Table 1 Characteristic values of the yarns drawn out from the fabric specimens.

\begin{tabular}{lllllll} 
& $\begin{array}{c}l_{0} \\
(\mathrm{~cm})\end{array}$ & $\begin{array}{c}l_{0} \\
(\mathrm{~cm})\end{array}$ & $\begin{array}{c}l_{0} \\
(\mathrm{~cm})\end{array}$ & $l_{c}$ & $\begin{array}{c}\mathrm{d} \\
\left(10^{-3} \mathrm{~cm}\right)\end{array}$ & $\mathrm{N}$ \\
\hline $\mathrm{A} 1$ & 5.0 & 5.04 & 5.73 & 5.57 & 1.87 & 96 \\
$\mathrm{~A} 2$ & 5.0 & 4.99 & 5.51 & 5.27 & 1.80 & 64 \\
$\mathrm{~B} 1$ & 5.0 & 5.05 & 6.01 & 6.40 & 2.39 & 32 \\
$\mathrm{~B} 2$ & 5.0 & 4.98 & 5.58 & 5.60 & 2.28 & 64 \\
$\mathrm{C} 1$ & 5.0 & 4.98 & 5.67 & 5.84 & 1.97 & 94 \\
$\mathrm{C} 2$ & 5.0 & 4.99 & 5.92 & 6.10 & 1.92 & 94
\end{tabular}

\subsection{Lateral Compressional Property of Yarns Drawn out} from the Fabric

The lateral compressional property of yarns was measured by the two-plate method (Fig. 6) in the past, but recently Kawabata et al. developed the channel method ${ }^{[6]}$ and the wire method ${ }^{[7]}$, and showed that the wire method is the most appropriate method for substituting the yarn compressional property into the biaxial extension theory of weaves.

We use here these measuring methods and compared the results as shown in Fig. 7. The data shown in Fig. 7 are averaged by the following equation, where $\phi_{1}$ and $\phi_{2}$ are the thickness decreases of the warp and the weft respectively by the lateral compression:

$$
h_{c}\left(f_{c}\right)=\frac{1}{2}\left\{\phi_{1}\left(f_{c}\right)+\phi_{2}\left(f_{c}\right)\right\}
$$

where $h_{c}\left(f_{c}\right)$ is the amount of decrease of yarn thickness as a function of the lateral compression force $f_{c}$. The 0 -point on the curve measured by the wire method is the starting point for the calculation. In the cases of the two-plate method and the channel method, an empirical adjustment of the data is necessary to substitute the data obtained by these methods into the biaxial extension theory of weaves. ${ }^{[1,6]}$ 


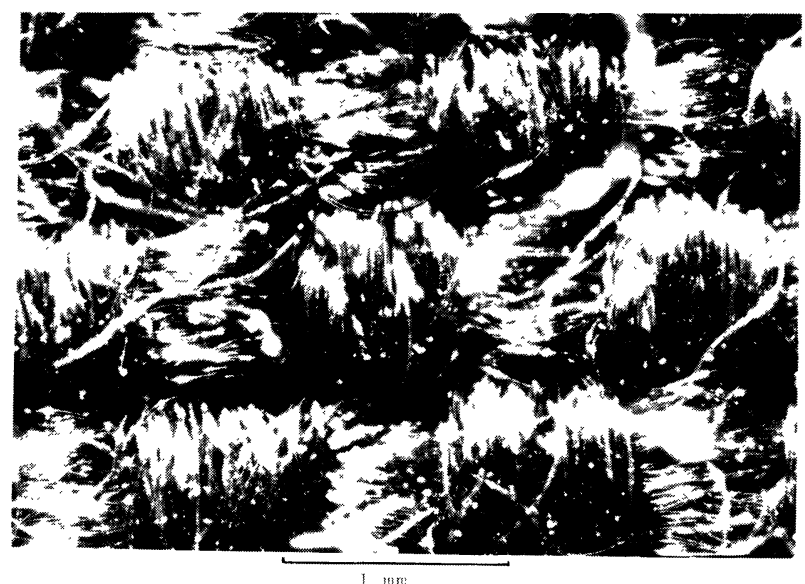

(a) Fabric $\mathrm{A}$

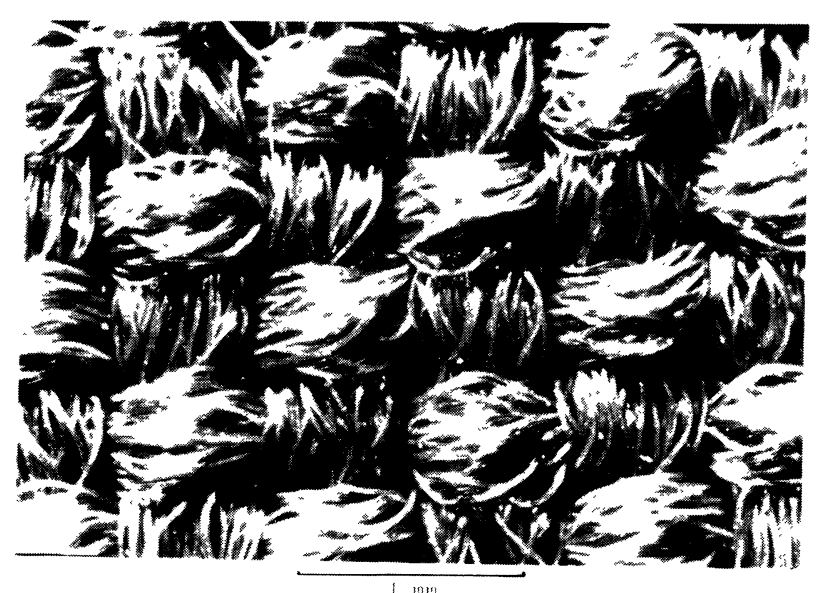

(b) Fabric B

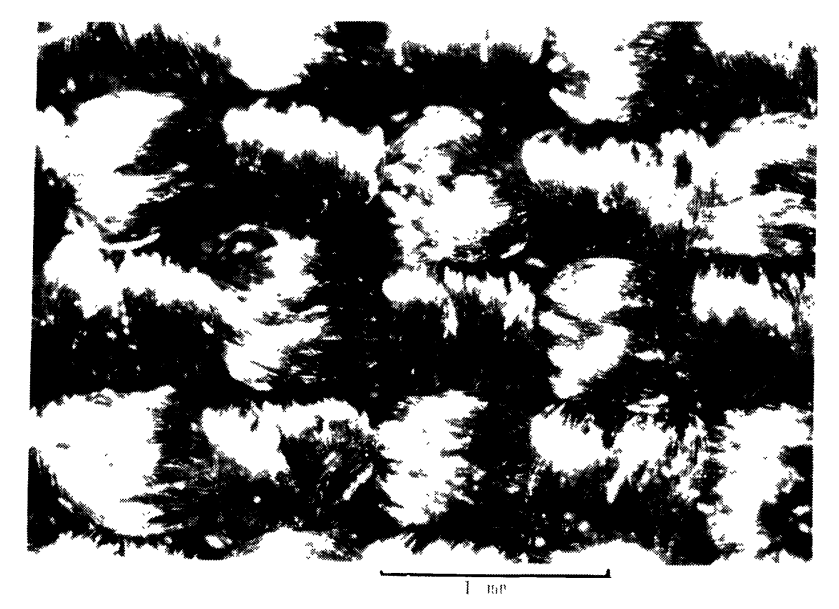

(c) Fabric C

Fig. 3 Photographs of the fabric surface.

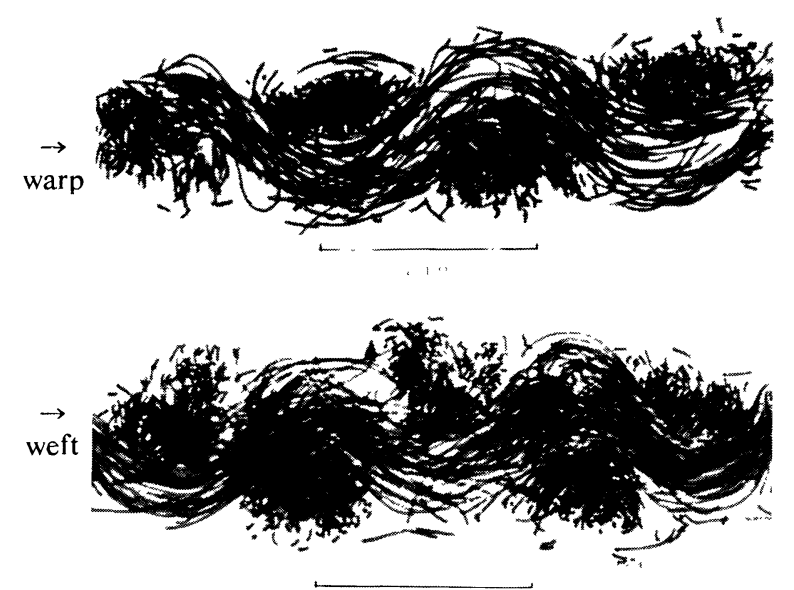

(a) Fabric A
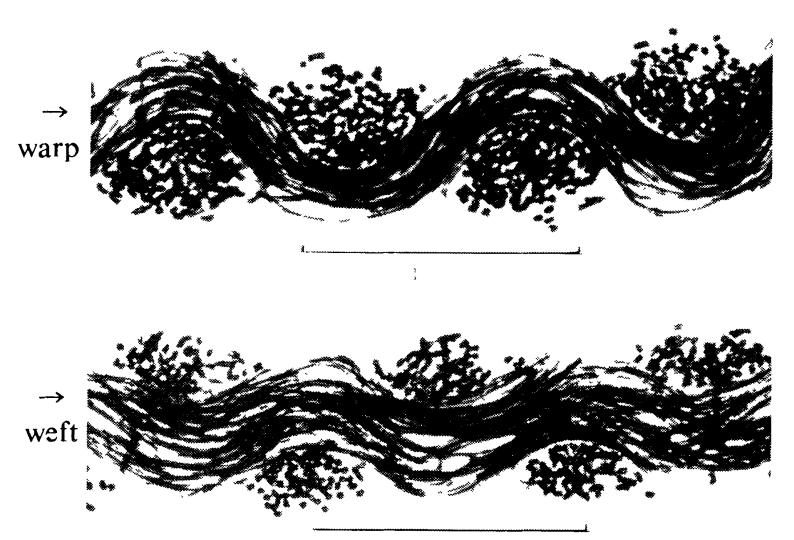

(b) Fabric B
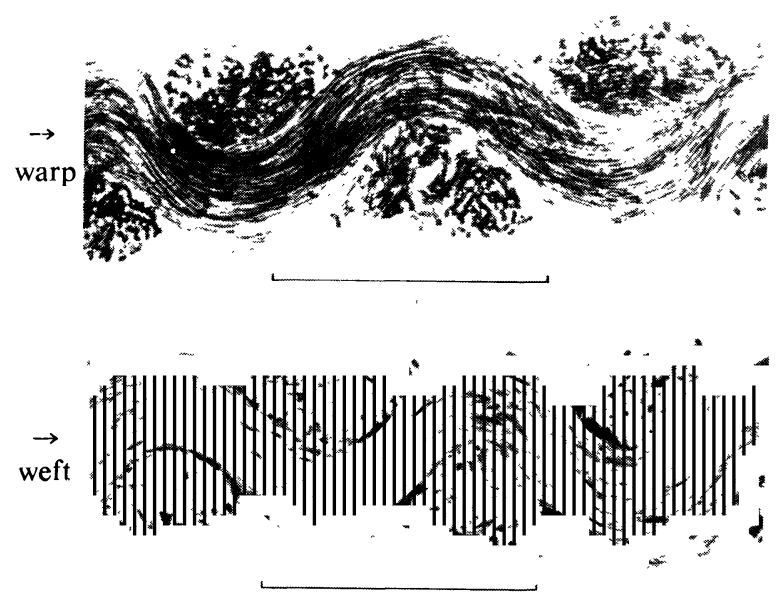

(c) Fabric C

Fig. 4 The cross-sections of fabrics.

Table 2 The yarn density of the fabric specimens $(\mathrm{l} / \mathrm{cm})$

$\begin{array}{llllll}\text { A1 } & \text { A2 } & \text { B1 } & \text { B2 } & \text { C1 } & \text { C2 } \\ 26.2 & 29.3 & 41.2 & 31.2 & 33.1 & 28.8\end{array}$




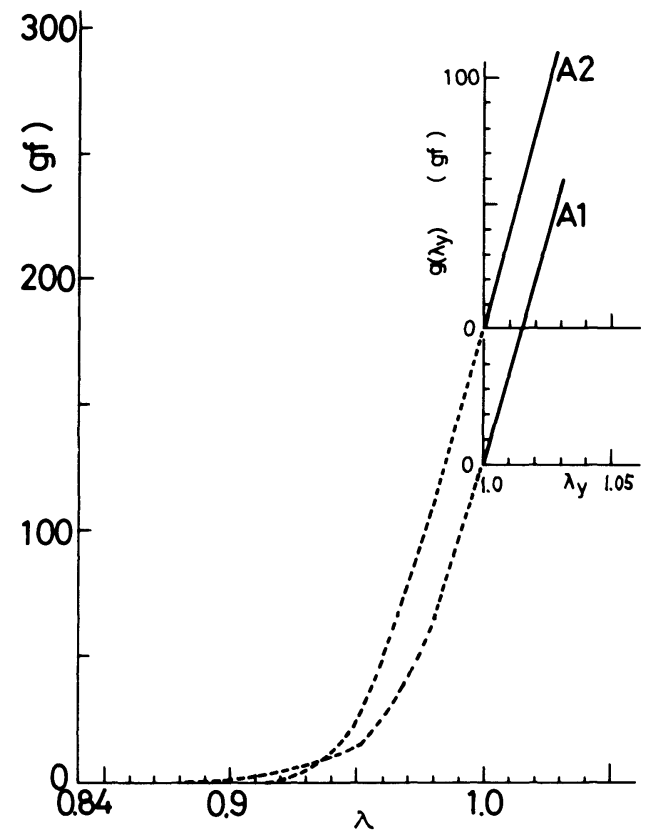

(a) Specimen A

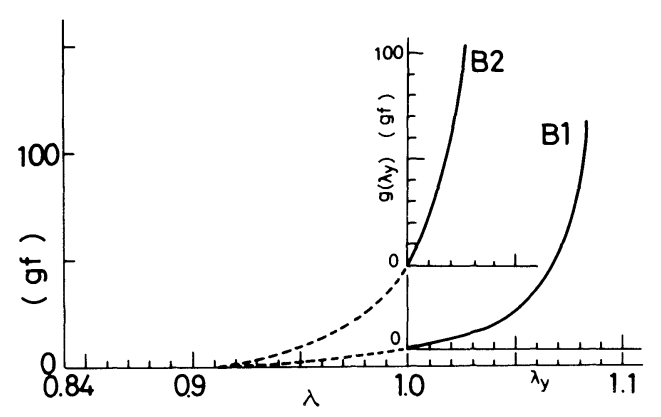

(b) Specimen B

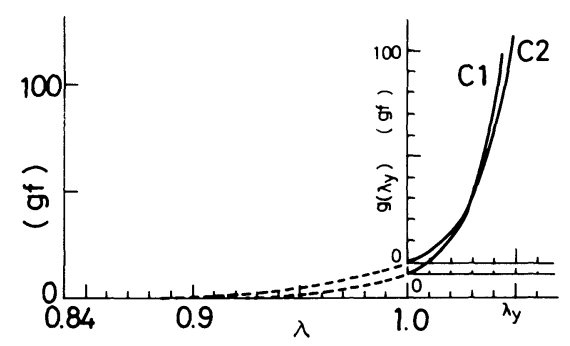

(c) Specimen $C$

Fig. 5 Measured tensile properties of yarn specimens drawn out from fabrics. The points 0 are the predicted initial extension states. The solid lines are used for the calculation.
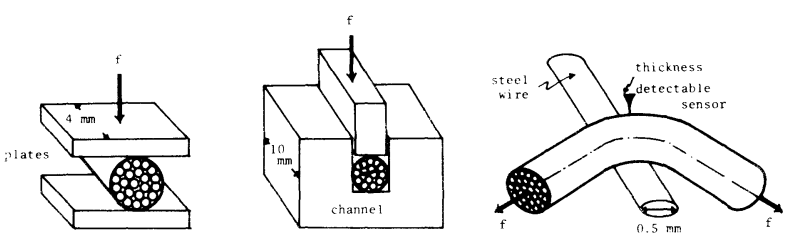

(b) channel method

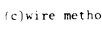

Fig. 6 Three types of measuring methods of lateral compression of yarns.

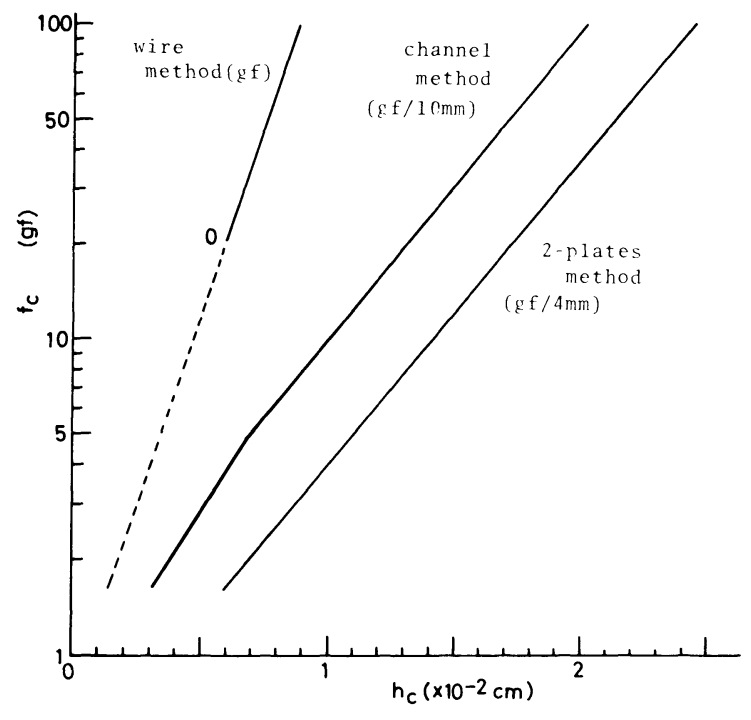

(a) Specimen $\mathrm{A}$

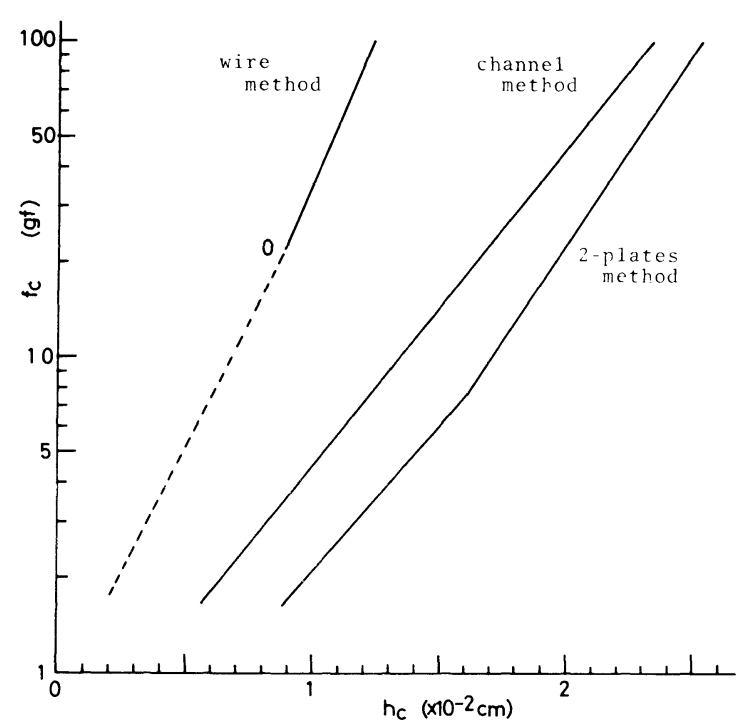

(b) Specimen B

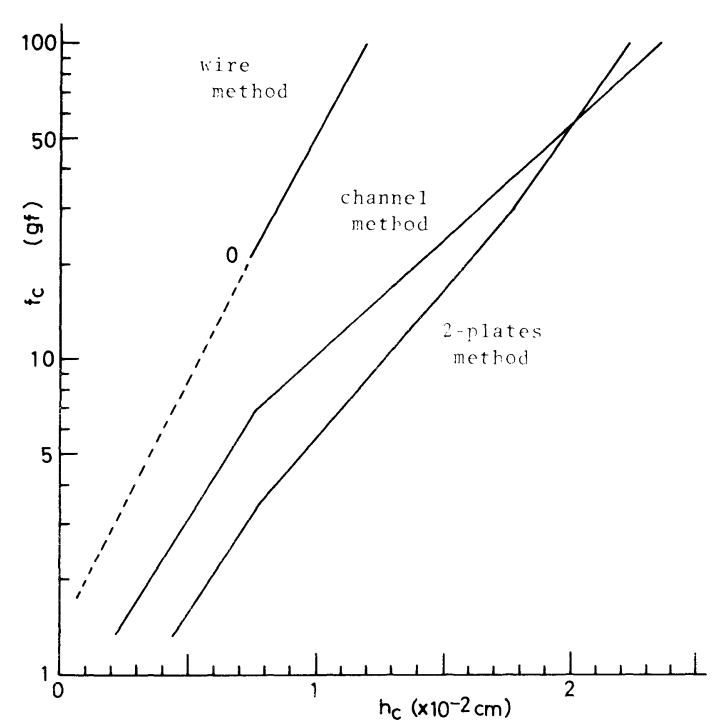

(c) Specimen $\mathrm{C}$

Fig. 7 Measured compressional properties of yarns drawn out from fabrics. Three methods were used. 


\subsection{Biaxial Tensile Properties of Plain Weave Fabrics}

We can now calculate the biaxial tensile properties of fabrics substituting these yarn properties into the theory of biaxial tensile property of fabric.

The compressional force $F_{c}$ at the interlacing point of yarns in fabrics is obtained as following, ${ }^{[1]}$

$$
\begin{aligned}
& F_{c 1}=2 g_{1}(\lambda y) \frac{2\left(h_{m 1}-h_{1}\right)}{\sqrt{4\left(h_{m 1}-h_{1}\right)^{2}+\left(\lambda_{y} y_{01}\right)^{2}}} \cdots \cdots \cdots(6) \\
& F_{c 2}=2 g_{2}(\lambda y) \frac{2\left(h_{m 2}+h_{2}\right)}{\sqrt{4\left(h_{m 2}+h_{2}\right)^{2}+\left(\lambda_{y} y_{02}\right)^{2}}} \cdots \cdots \cdots(7)
\end{aligned}
$$

and $F_{\mathrm{c}}=F_{\mathrm{c} 1}=F_{\mathrm{c} 2}$

Now, $F_{c 1}$ and $F_{c 2}$ are obtained as the functions of $h_{1}$ and $h_{2}$ for given $\lambda_{1}$ and $\lambda_{2}$, where

$g\left(\lambda_{y}\right)$ : tensile property of yarn

$h$ : distance between the weave neutral line and the yarn axis along the $\mathrm{X}_{3}$-axis, ${ }^{[1]}$ and this distance changes by the stretching

$h_{m}$ : distance between the neutral line and the yarn axis along the $\mathrm{X}_{3}$-axis in the unstretched state of fabric; this is also equal to the maximum value of $h$

$\lambda_{y}$ : stretch ratio of the yarn, where the stretch ratio $\lambda$ is defined by $\lambda=1+$ strain

$y_{0}$ : thread-spacing in undeformed state

$n$ : yarn density in the undeformed state (number of ends/ cm) $\left(n_{1}=1 / y_{02}\right)$

Suffices 1 and 2 mean the warp and the weft respectively. The values shown in Table 1 are aso used to calculate the compressional force $F_{c}$. In the case of incompressible yarn, $h_{1}=$ $h_{2}$ and $h_{1}$ can be solved for given $\lambda_{1}$ and $\lambda_{2}$.

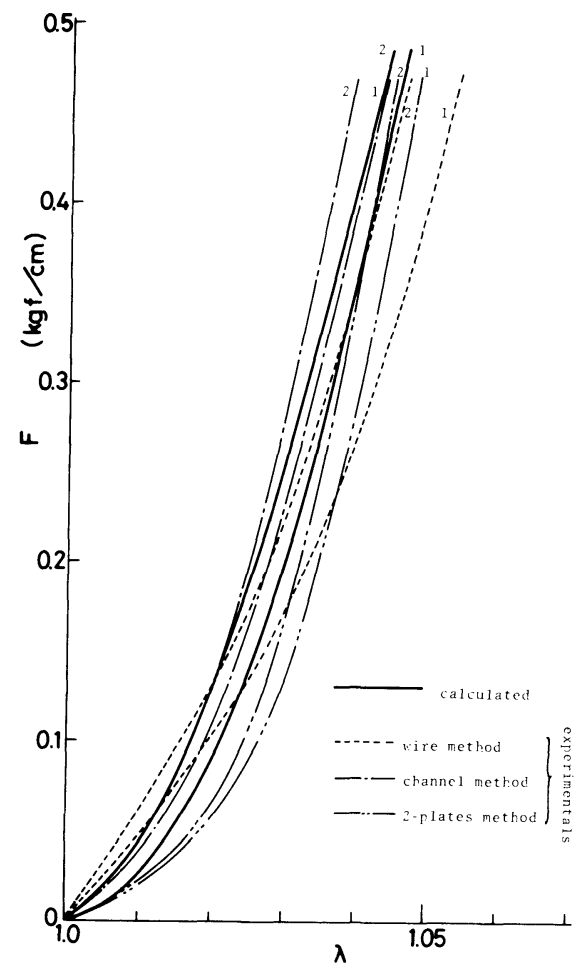

(a) Specimen $\mathrm{A}$

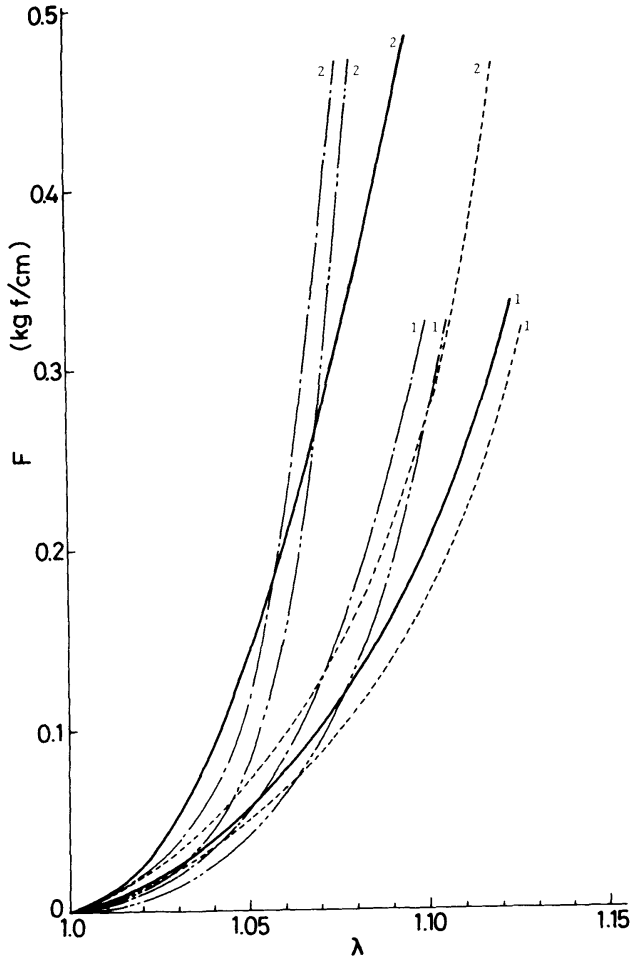

(b) Specimen B

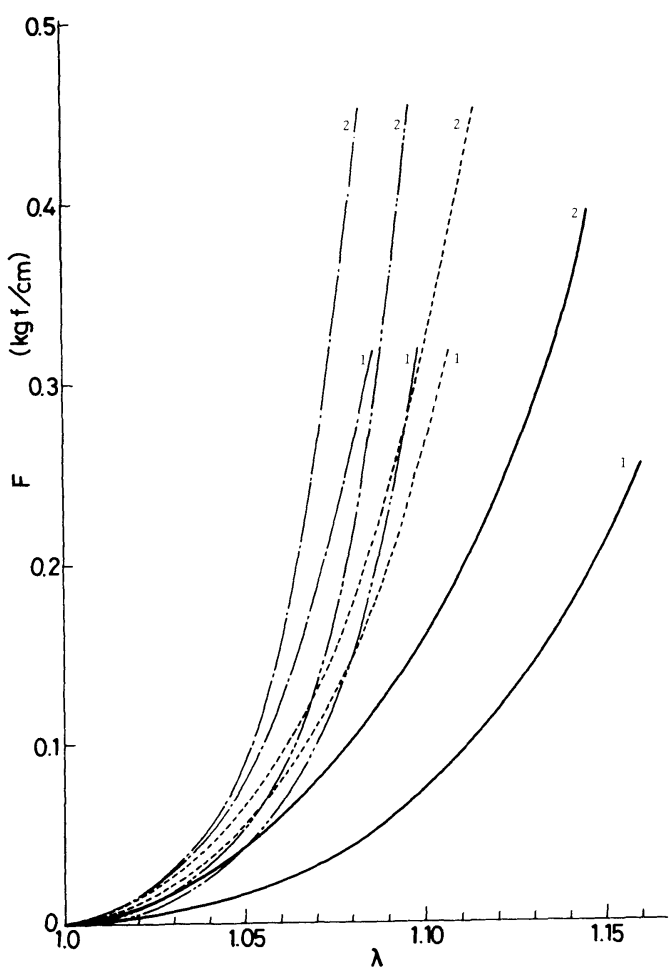

(c) Specimen $\mathrm{C}$

Fig. 8 Biaxial tensile properties of fabric specimens when $\lambda_{1}=$ $\lambda_{2}=\lambda$.

For introducing the compressibility of yarn in the equations (6) to (8), the $h_{2}$ in these equations is replaced by

$$
h_{2}\left(F_{c}\right)=h_{1}\left(F_{c}\right)-h_{c}\left(F_{c}\right.
$$

The biaxial tensile forces $F_{1}$ (along the warp) and $F_{2}$ (along the weft) at given extension ratios $\lambda_{1}$ and $\lambda_{2}$ of the fabric are obtained from 


$$
F_{1}=\frac{F_{c} \cdot \lambda_{1} \cdot y_{01}}{4\left(h_{m 1}-h_{1}\right)}
$$

and

$$
F_{2}=\frac{F_{c} \cdot \lambda_{2} \cdot y_{02}}{4\left(h_{m 2}+\widetilde{h}_{2}\right)}
$$

where $\widetilde{h}_{1}$ and $\widetilde{h}_{2}$ are the solutions of equation (8) for given $\lambda_{1}$ and $\lambda_{2}$.

We measured the biaxial tensile properties at the condition $\lambda_{1}=\lambda_{2}$ (equal biaxial extension) for samples A, B and $\mathrm{C}$, and the results are shown in Fig. 8 together with the calculated results made by using the yarn compressional properties by the three methods. The experimentals and the calculated results show better agreements for specimens $\mathrm{A}$ and $\mathrm{B}$ rather than for specimen $\mathrm{C}$ in the deformation range below $500 \mathrm{gf} / \mathrm{cm}$.

\section{Discussions}

In this experiment, the maximum load for the biaxial fabric extension is $500 \mathrm{gf} / \mathrm{cm}$, which is rather low for fabric stretch. In the case of specimen $\mathrm{C}$, there was a considerable difference between experimentals and calculated results. The reason of the difference may be caused by the error in the measurement of yarn compressional property where the yarns in the fabric is relatively stiff in bending compared with the other yarns in fabrics.

We have examined the channel and the wire methods in addition to the two-plate method to investigate which method is adequate. As the lateral compressional property of a yarn by the wire method simulates the lateral compression of yarns in the fabric due to the extension of yarns in the fabric, so the compressional property obtained by the wire method is very similar to the actual state of the yarn in the fabric. Thus, we can introduce the compressional property as it is to the biaxial tensile theory of fabrics when we use the compressional properties measured by the wire method.

Finally, we think that the experiments must be measured more precisely, and the results must be discussed furthermore after this.

\section{Conclusions}

(1) In order to calculate the biaxal tensile properties of textured yarn fabrics applying the biaxial tensile theory developed by Kawabata et al., we prescribed the initial extension state of yarns in the fabric before stretching, which is hitherto uncertain because of the stretchability of textured yarns.

(2) The agreement of experimental and calculated results is good for specimen, A, which is woven by ordinary flexible warp and weft yarns, but there was a difference for specimen C.

We express our thanks to Professor Masako Niwa, Nara Women's University, for her support to our experiments, and to Miss Sachiko Sukigara, graduate student there, for her help to our calculations.

\section{References}

[1] S. Kawabata, M. Niwa, H. Kawai; J. Text. Inst., 64, 21 (1973).

[2] S. Kawabata, M. Niwa; J. Text. Inst., 70, 417 (1979).

[3] W. D. Freeston, Jr., M. M. Platt, M. M. Schoppee; Text. Res. J., 37, 948 (1967).

[4] S. Kawabata, T. Sasai; J. Text. Mach. Soc. Japan, 29, T197 (1976).

[5] S. Kawabata, T. Sasai; J. Text. Mach. Soc. Japan, 29, T140 (1976).

[6] S. Kawabata, M. Niwa, Y. Kawai; J. Text. Mach. Soc. Japan, 31, T74 (1978).

[7] S. Kawabata, M. Niwa, M. Matsudaira; J. Text. Mach. Soc. Japan, 34, T33 (1981). 